\title{
GALACTIC ORBITS OF DIFFERENT GALACTIC POPULATION OBJECTS
}

\author{
L. P. OSSIPKOV AND S. A. KUTUZOV \\ St Petersburg State University, \\ Stary Peterhof, St Petersburg 198904, Russia
}

1. Introduction. It is evident that the knowledge of not only positions and velocities of objects belonging to different populations but also their osculating galactic orbits can clarify the structure and history of our Galaxy. Action variables being adiabatic invariants are especially useful for these purposes ( Binney, May 1986). One of them is a usual area integral (and it can be found without any orbit calculation ). The second action variable is connected with an energy integral but for 3-D orbits and arbitrary potentials it cannot be found analitically. Eccentricities resulting from orbit calculations can be used here ( Lynden-Bell 1963 ). Comparison of eccentricities defined with different ways shown that differences between them are practically negligible ( Kutuzov 1985, 1987 ). As for the third action variable and the corresponding third isolating integral orbit calculations can confirm its existence and control the validity of various approximations. Numerical technique allowing to find it and working at any potential is now in progress ( Binney, Kumar 1993 ).

2. Galactic potential and orbital elements. We used the axially symmetrical two-component model of our Galaxy ( Kutuzov, Ossipkov 1989 ) for orbit calculations. Pericenter and apocenter cylindrical distances $R_{p}$ and $R_{a}$ and the maximal height of objects over the galactic plane $z_{m}$ were used as orbital elements and were finding in course of orbit calculations. Two dimensionless quantities $e=\left(R_{a}-R_{p}\right) /\left(R_{a}+R_{p}\right), c=$ $2 z_{m} /\left(R_{a}-R_{p}\right)$ and one dimensional quantity, $R_{p}$, were considered instead of them. Sometimes we preferred to find spherical distances $r_{a}$ and $r_{p}$ and spherical eccentricity $e_{s}=\left(r_{a}-r_{p}\right) /\left(r_{a}+r_{p}\right)$.

3. Results. 3.1. Open clusters. All orbits were found to be box ones, and the boxes were practically rectangles. The conservation of the adiabatic invariant of vertical motion $J_{z}=\left[V_{z}^{2}+C^{2}(R) z^{2}\right] /[2 C(R)], \quad C^{2}(R)=$ $-\partial^{2} \Phi(R, z) /\left.\partial z^{2}\right|_{z=0}$ ( Ossipkov 1976, Villumsen, Binney 1985 ) has been 
checked. We found that if $J_{z}<1(\mathrm{~km} / \mathrm{s}) / \mathrm{kpc}$ then changes of $J_{z}$ do not exceed 0.01 . Mean values of orbital elements $e, c, z_{m}$ are equal to to $0.06 \pm 0.01,0.50 \pm 0.06,0.21 \pm 0.03 \mathrm{kpc}$ respectively.

3.2. Planetary nebulae. We found that some orbits do not fill rectangle boxes and sometimes they are practically hyperbolic. Mean values of orbital elements are $\langle e\rangle=(0.11 ; 0.16),\langle c\rangle=(0.40 ; 0.81),\left\langle z_{m}\right\rangle=(0.36 ; 0.54)$ kpc. The first values are given for Khromov's distances (1985) and the second values correspond to Cudworth's ones (1974).

3.3. Short-periodic variables. We compiled data on proper motions and radial velocities for 76 short-periodic stars. Sources used by us are given by Ossipkov, Kutuzov (1993). For RRa type stars with the period $P<0 .^{d} 4$ we found $\left\langle R_{p}\right\rangle=6.65 \mathrm{kpc},\langle e\rangle=0.26,\left\langle z_{m}\right\rangle=1.68 \mathrm{kpc}$. For stars with $0 .{ }^{d} 4<P<0 .^{d} 6$ we have $\left\langle R_{p}\right\rangle=4.43 \mathrm{kpc},\left\langle e>=0.48,\left\langle z_{m}\right\rangle=\right.$ $5.93 \mathrm{kpc}$. If $0 .{ }^{d} 5<P<0 .{ }^{d} 6$ then $\left\langle R_{p}\right\rangle=3.22 \mathrm{kpc},\langle e\rangle=0.56,\left\langle z_{m}\right\rangle=$ $6.38 \mathrm{kpc}$. And for stars with $P>0 .^{d} 6<R_{p}>=2.90 \mathrm{kpc},<e>=0.48,<$ $z_{m}>=6.80 \mathrm{kpc}$. For RRc variables $\left\langle R_{p}\right\rangle=2.78 \mathrm{kpc},\langle e>=0.63,<$ $z_{m}>=6.58 \mathrm{kpc}$.

3.4. Globular clusters. New proper motions of globular clusters compiled by Allen, Santillan (1993) and Cudworth, Hanson (1993) were used for orbit calculations of these objects. There are some evidences of existence of three globular cluster groups forming parallel sequences on the metallicity - eccentricity diagram. Critical values of metallicity are -1.4 and -2.0 .

3.5. High-velocity metal-poor stars. We have used data compiled by Allen et al. (1991) for orbit calculations for 40 high-velocity stars with $[\mathrm{Fe} / \mathrm{H}]<-2.0$. The sample was found to be extremely non-uniform. Orbits of 4 stars have $e_{s}<0.2$ and 10 stars have $e_{s}>0.9$.

\section{References}

Allen, C., Santillan, A. (1993) Rev.Mex.Astron.Astrof., Vol. 25, p.39.

Allen, C., Schuster, W., Poveda, A. (1991) AA, Vol. 244, p. 286.

Binney, J., May, A. (1986) $M N$, Vol. 218, p. 743.

Binney, J., Kumar, S. (1993) $M N$, Vol. 261, p. 584.

Cudworth, K.M. (1974) $A J$, Vol. 80, p.199.

Cudworth, K.M., Hanson, R.M. (1993) AJ, Vol. 105, p. 168.

Khromov, G.S. (1985) Planetary Nebulae, Nauka, Moscow.

Kutuzov, S.A. (1985) Astronomical and Geodetical Studies, ed. K.A.Barkhatova. Ural Univ. Press, Sverdlovsk, p.56.

Kutuzov, S.A. (1987) Kin. i fiz. neb. tel, Vol.3, No 4, p.11.

Kutuzov, S.A., Ossipkov, L.P. (1989) $A Z h$, Vol. 66, p. 965.

Lynden-Bell, D. (1963) Observatory, No 932, p. 232.

Ossipkov, L.P. (1976) Pis'ma v AZh, Vol. 2, p. 367.

Ossipkov, L.P., Kutuzov, S.A. (1993) Galactic Bulges, ed. H.Dejonghe, H.J.Habing. Kluwer, Dordrecht, p.369.

Villumsen, J.V., Binney, J. (1985) ApJ, Vol. 295, p.388. 